\title{
Delivery Strategies for Skin: Comparison of Nanoliter Jets, Needles and Topical Solutions
}

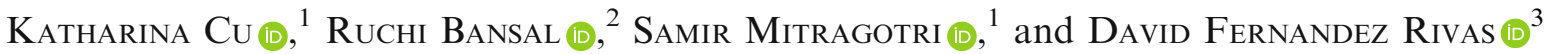 \\ ${ }^{1}$ John A. Paulson School of Engineering and Applied Sciences, Harvard University, Cambridge, MA, USA; ${ }^{2}$ Department of \\ Biomaterials Science and Technology, University of Twente, Enschede, The Netherlands; and ${ }^{3}$ Mesoscale Chemical Systems, \\ MESA + Institute, University of Twente, Enschede, The Netherlands
}

(Received 16 July 2019; accepted 9 October 2019; published online 15 October 2019)

Associate Editor Sriram Neelamegham oversaw the review of this article.

\begin{abstract}
Drug diffusion within the skin with a needle-free micro-jet injection (NFI) device was compared with two wellestablished delivery methods: topical application and solid needle injection. A permanent make-up (PMU) machine, normally used for dermal pigmentation, was utilized as a solid needle injection method. For NFIs a continuous wave (CW) laser diode was used to create a bubble inside a microfluidic device containing a light absorbing solution. Each method delivered two different solutions into ex vivo porcine skin. The first solution consisted of a red dye (direct red 81) and rhodamine B in water. The second solution was direct red 81 and rhodamine $\mathrm{B}$ in water and glycerol. We measured the diffusion depth, width and surface area of the solutions in all the injected skin samples. The NFI has a higher vertical dispersion velocity of $3 \times 10^{5} \mu \mathrm{m} / \mathrm{s}$ compared to topical $(0.1 \mu \mathrm{m} / \mathrm{s})$ and needle injection $(53 \mu \mathrm{m} / \mathrm{s})$. The limitations and advantages of each method are discussed, and we conclude that the micro-jet injector represents a fast and minimally invasive injection method, while the solid needle injector causes notable tissue damage. In contrast, the topical method had the slowest diffusion rate but causes no visible damage to the skin.
\end{abstract}

Keywords-Transdermal delivery, Needle-free injection, Thermocavitation, Solid needle injection, Topical application, Laser micro-jet.

\begin{tabular}{ll}
\multicolumn{2}{c}{ ABBREVIATIONS } \\
NFI & Needle-free injection \\
PMU & Permanent make-up \\
& \\
\hline \multicolumn{2}{c}{ Address correspondence to and David Fernandez Rivas, Me- } \\
soscale Chemical Systems, MESA + Institute, University of Twente, \\
$\begin{array}{l}\text { Enschede, The Netherlands. Electronic mail: d.fernandezrivas@ } \\
\text { utwente.nl }\end{array}$
\end{tabular}

$\begin{array}{ll}\text { CW } & \text { Continuous wave } \\ \mathrm{L}_{\mathrm{M}} & \text { Penetration depth } \\ \mathrm{L}_{\mathrm{T}} & \text { Penetration width }\end{array}$

\section{INTRODUCTION}

For many centuries, needles and syringes have been extensively used in several medical procedures. And for just as long, injections are feared by many patients. ${ }^{2,38,39}$ Pills or topical skin products are easier to use and are painless. Accordingly, oral and transdermal administration routes are favored by patients

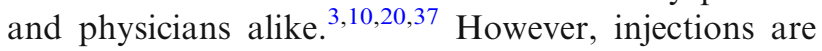
difficult to replace since certain drugs can be administered via intramuscular, subcutaneous or intravenous injections, where drugs reach the systemic circulation with high efficiency. Topically applied drugs via creams or patches exhibit slow drug uptake due to the passive delivery across the skin induced by a concentration gradient, in which the diffusion properties are a function of the skin characteristics and the solution molecules. ${ }^{38}$ The slow diffusion originates primarily from the properties of the outermost skin layer, the stratum corneum (SC), which protects the underlying tissue from infections and dehydration. ${ }^{30}$ In practice, diffusion is only limited to lipophilic and low molecular weight drugs $(<500 \mathrm{~g} / \mathrm{mol}){ }^{5,10,38}$ Thus, the SC poses a great permeation challenge for most of the drugs and delivery methods since the majority has a high molecular weight and poor solubility. ${ }^{6}$ One alternative to the topical application is the use of microneedles, which are effective for breaking through 
the SC but have poor accuracy of delivery, among other limitations. ${ }^{24}$

Other injection alternatives developed over the last decades, such as high-pressure injections and needleassisted jet injectors, have shown advantages of needlefree injections (NFIs), in which a pressurized liquid (or powder) jet penetrates the skin. ${ }^{4,21,26,31,32,47}$ Infections as well as disease transmissions due to improper (re)use of needles are irrelevant when the disposal of needles is not required, which also reduces high costs for singleuse components. ${ }^{5,12}$ The most important advantage of an NFI device is thought to be pain reduction that results in higher patient compliance, especially in chronic diseases like diabetes or by people fearing sharps. ${ }^{17,18}$ From the different energy sources used to power NFIs, a recent example is based on continuous wave $(\mathrm{CW})$ lasers that cause a phenomenon known as thermocavitation to create liquid jets by heating the injectate above its boiling point with an explosive phase transition. . $^{8,33,35,42}$ Until now, jets made with $\mathrm{CW}$ lasers exhibit more modest velocity values $(\sim 40 \mathrm{~m} / \mathrm{s})$ than those achieved with other injector concepts, e.g. pulsed lasers $(850 \mathrm{~m} / \mathrm{s})$ and voice coils $(100 \mathrm{~m} / \mathrm{s}) .{ }^{22,28,48,49}$ However, as it is evidenced in this study, thermocavitation jets seem to perfuse ex vivo porcine skin. Since this method is still in its infancy, no reliable studies have been performed on the potential physicochemical changes that the heating of the liquid might induce. A solution to avoid injecting degraded liquid has been proposed, in which an impermeable membrane separates the injectate from the heated liquid. ${ }^{19}$

This work evaluates a CW-based needle-free microjet injector as a possible transdermal delivery alternative with minimal damage to the skin structure. For the purpose of this study, the topical solution delivery will be primarily associated with diffusion processes. In terms of delivery by the penetrating solid needle or liquid jet and subsequent diffusion, we will refer to this process as penetration or perfusion. The solid needle actuated by a medical dermal pigmentation device was chosen due to its use for small volume delivery, to be compared with the micro-jet method introduced supra. $^{21,33}$ The combined diffusion and penetration processes are defined as total dispersion. We investigated the potential for $\mathrm{CW}$-induced jet injection to achieve deeper dispersion depths than with topical application or solid needle injections. All three methods had different injection or application durations but were compared after $60 \mathrm{~min}$ for analysis. The metrics for comparison used in this work are the diffusion and dispersion depths, widths and surface areas for the topically applied or injected solutions, and the evaluation of the spread of the drug within the skin, characterized by the dispersion distances.

\section{MATERIALS AND METHODS}

Three different types of experiments were performed with porcine skin: one topical and two injection experiments. For the first injection experiment, a permanent make-up (PMU) machine was used as solid needle injector. The other was a needle-free micro-jet injector based on thermocavitation. For all methods, excess solution was carefully removed from the skin with a Kimwipe paper tissue (Kimberly-Clark).

\section{Ex Vivo Porcine Skin}

Ex vivo abdominal porcine skin tissue was procured from the abattoir (Slagerij Nijboer, Enschede, the Netherlands). Since the skin properties vary from animal to animal, all experiments and, replicates were made with the exact same porcine skin sheet to ensure a fair comparison of results. While performing the experiments, the remaining fresh porcine skin samples were stored at $4{ }^{\circ} \mathrm{C}$ in Dulbecco's Modified Eagle Medium (DMEM, Sigma-Aldrich). A surgical knife was used to remove excess fat tissue and to cut the skin samples into $3 \times 3 \mathrm{~cm}$ sizes, with a thickness ranging between 2 and $3 \mathrm{~mm}$. The fat was removed to minimize potential errors in the determination of the lateral injectate dispersion. To emulate real-life scenarios, and compensate for the fat removed, 5\% agarose slabs were placed under the skin to still mimic the global skin stiffness. ${ }^{29,46,48}$ The skin samples were carefully dried with a Kimwipe before each experiment to prevent solution running. On average, all samples were used for experimentation within $2 \mathrm{~h}$ after obtaining them from the abattoir.

\section{Liquid Solutions}

Two different solutions were used in all experiments. The aqueous solution consisted of $0.15 \mathrm{wt} \%$ rhodamine B (Sigma-Aldrich) and $0.25 \%$ wt direct red 81 (Sigma-Aldrich). While rhodamine B was used for its fluorescing properties, the direct red 81 was needed to maximize the laser energy absorption for the needlefree micro-jet injector. For the glycerol solution, 10\% glycerol (Sigma-Aldrich) was added. Unlike other studies that used glycerol for its viscosity increasing properties, we used it as diffusion enhancer due to its use in the pharmaceutical industry as a moisturizer. ${ }^{16}$ As measured elsewhere, ${ }^{46}$ both solutions behave as Newtonian fluids with measured constant viscosity $\eta_{\text {aqueous }}=0.9 \mathrm{mPa} . \mathrm{s}$ and $\eta_{\mathrm{glyc} 10 \%}=1.2 \mathrm{mPa} . \mathrm{s} .{ }^{33} \mathrm{The}$ absorption was measured for both solutions in the spectral range of (300-1000) nm (UV-Visible Spectrophotometer UV-2600, Shimadzu). 


\section{Topical Application}

A pipette (Eppendorf) was used to apply $2 \mu$ solution drops onto the skin surface. Dye diffusion was compared at three different time intervals (after 5, 30 and $60 \mathrm{~min}$ ). Once the time elapsed, any excess solution was carefully removed with a Kimwipe before being frozen in 2-methyl butane (Sigma-Aldrich). Each experiment was repeated five times, which resulted in 30 samples for the two different solutions.

\section{Solid Needle Injector}

The handpiece of the PMU machine (PL-1000 Mobil, Permanent Line GmbH) was vertically placed in a custom-made holder to inject various solutions into porcine skin, as shown in Fig. 1. The encased motor in the handpiece smoothly moves the solid needle within the attached cartridge in a cyclic pattern up and down. ${ }^{23}$ With the electrical control unit of the PMU machine, the frequency of the needle sinusoidal movement in the vertical direction is adjustable in the range of $f=(50-150) \mathrm{Hz}$. The disposable needle cartridge contains a sterilized, stainless-steel needle with a diameter of $\mathrm{d}_{\text {Needle }}=0.4 \mathrm{~mm} \quad$ (Permanent Line $\mathrm{GmbH})$. For the injection procedure, a syringe was used to dispense $2 \mu$ lof the solution into the cartridge orifice. The needle was fully retracted and placed at $1 \mathrm{~mm}$ distance from the porcine skin samples, which were fixed by an in-house 3D printed case.

To mimic the underlying muscle and fat tissue, $5 \mathrm{wt} \%$ agarose (Sigma-Aldrich) was prepared and poured into the 3D-printed holder, which elastic modulus was measured elsewhere to be $17 \mathrm{MPa} .^{34}$ After the agarose solidified, the cut porcine skin samples were placed on top of it. Two lids held the skin

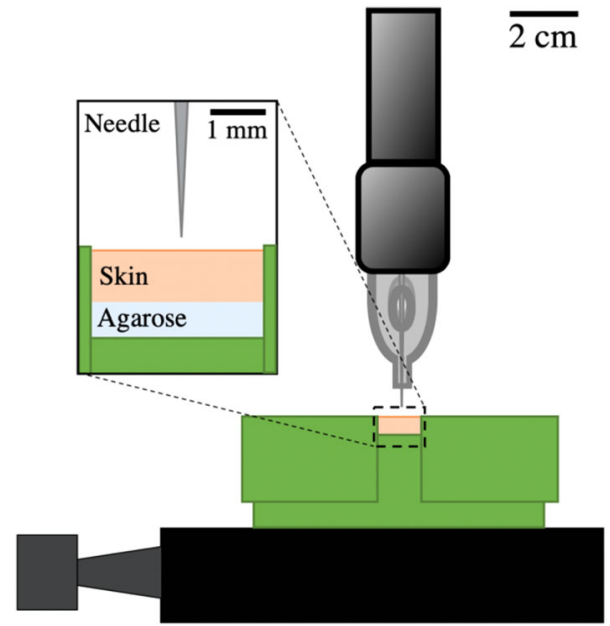

FIGURE 1. Schematic setup of solid needle injector. PMU machine hand-piece held vertically by custom-made holder for injections into porcine skin, which was fixed by 3D printed case. tight, while the needle penetrated the skin at a frequency of $100 \mathrm{~Hz}$. The vertical load placed onto the skin samples is challenging to quantify due to the difficulties of sample fixation. The vertical load, however, is assumed to be equal for all samples. The injections of $20 \mathrm{~s}$ were timed with a circuit and an Arduino code, which turned on the PMU machine for the respective time. Five replicates were made for each solution, which in total resulted in ten porcine skin samples.

\section{Needle-Free Micro-Jet Injector}

The experimental setup for the needle-free micro-jet injector consists of a CW laser diode $(\lambda=450 \mathrm{~nm})$ and a microfluidic device composed of two anodically bonded borosilicate glass wafers (Schott AG). The fabrication process and design of these devices were previously described elsewhere. ${ }^{8,9}$ The microfluidic devices consisted of two rectangular channels with $100 \mu \mathrm{m}$ depth: one for the cavitation bubble formation and the second one for the liquid jet to exit. An inlet of the same depth controlled the volume into the first channel $(500 \mu \mathrm{m}$ height, $1800 \mu \mathrm{m}$ length). The laser beam was focused with a $\times 10$ microscope objective at the bottom of the device, which was fixed with its holder to an XYZ linear translation stage holder to align the device with respect to the focused laser spot, as seen in Fig. 2. Next, the first channel of the device was partly filled with one of the solutions by manually positioning the meniscus with a syringe to a channel position of $500 \mu \mathrm{m}$. A high-speed camera (Fastcam SA-X2, Photron) was then simultaneously triggered with a circuit and an Arduino code, which turned on the laser $(U=4.7 \mathrm{~V}, P=1.2 \mathrm{~W}$ ) for $100 \mathrm{~ms}$ to record all experiments at $160 \times 10^{3} \mathrm{frames} / \mathrm{s}$ (fps). Within a few microseconds, the liquid inside the partially filled device was heated up above its boiling point. As a result, a fast-growing vapor bubble was created at the beam spot, a phenomenon known as thermocavitation. ${ }^{35,42}$ The growing bubble simultaneously pushes the liquid out of the first channel and forms an injection jet. A white light source was positioned on the opposite of the high-speed camera to visualize the jet propagation (velocity and shape) and penetration into

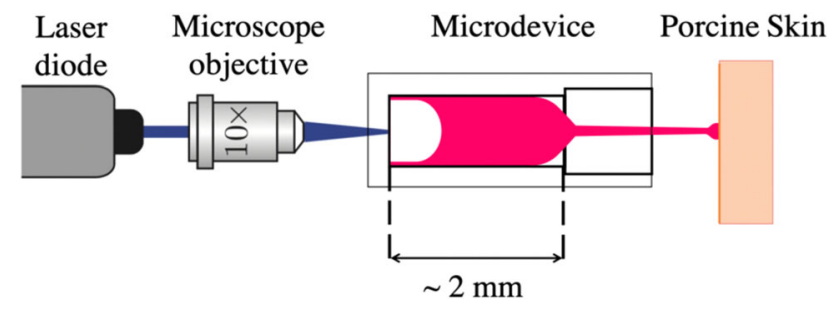

FIGURE 2. Schematic setup of the needle-free micro-jet injector. A microscope objective was used to focus the laser at the bottom of the microfluidic device. With a high-speed camera the bubble and jet formation, as well as skin penetration were recorded. 
(A)
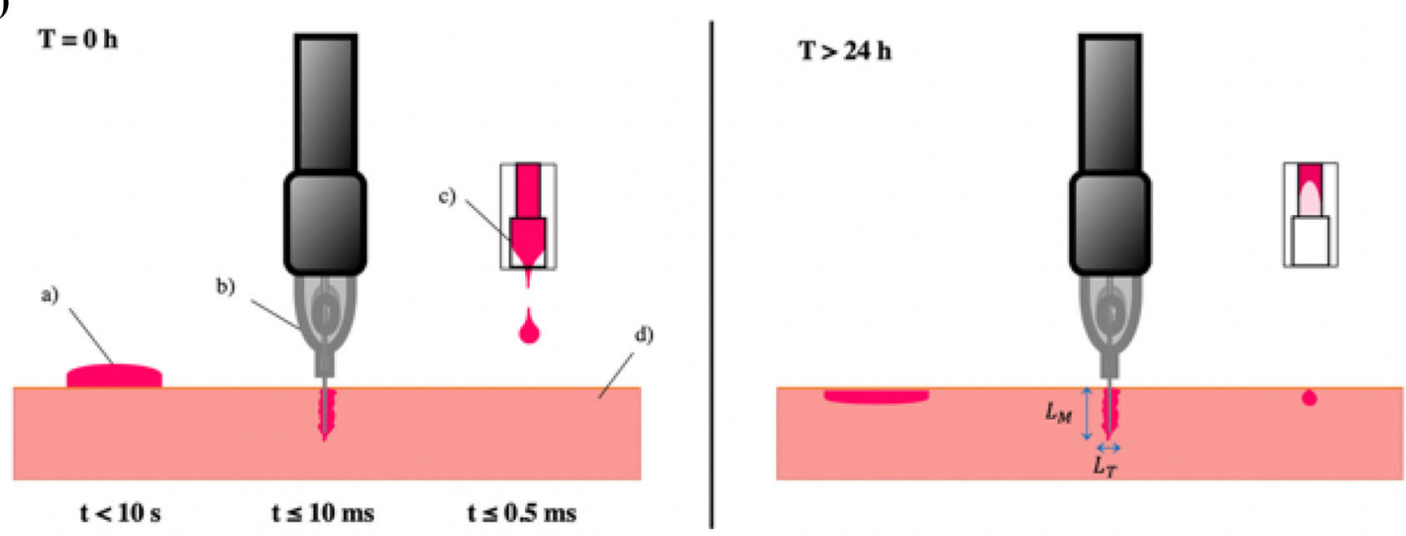

(B)
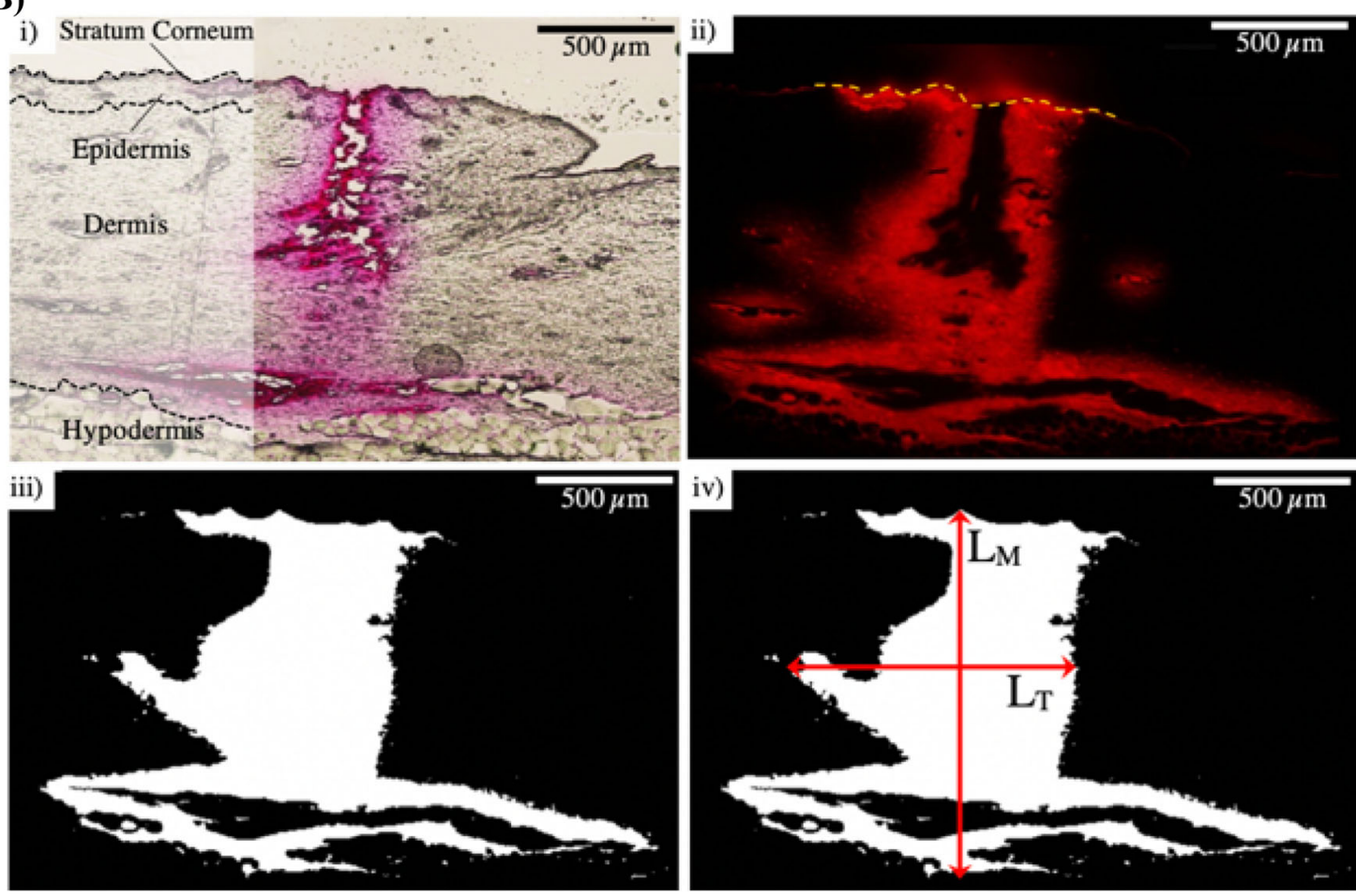

FIGURE 3. (A) Injection phases. Left panel: Instantaneous application or injection takes < $10 \mathrm{~s}$ for (a) topical application and (b) $<10 \mathrm{~ms}$ for solid needle injection or (c) $0.5 \mathrm{~ms}$ for micro-jet injection. Right panel: Solution dispersion throughout the skin, $24 \mathrm{~h}$ after application/injection; (d) porcine skin with the stratum corneum; (B) Image processing analysis. (i) Bright-field image of a solid needle injection and schematic drawing of different skin layers showing the stratum corneum on top. Glycerol solution was injected for $20 \mathrm{~s}$ at $100 \mathrm{~Hz}$; (ii) Respective fluorescence image; the yellow dotted line indicates the porcine skin surface; (iii) Calculated surface area of glycerol solution injection represented in white after image processing (101 $\left.\times 10^{4} \mu \mathrm{m}^{2}\right)$; (iv) Diffusion depth $L_{M}$ and width $L_{T}$ measurements with ImageJ. Scale bars correspond to $500 \mu \mathrm{m}$.

the skin samples. The jet velocities $v_{\text {jet }}$ were calculated out of the image sequences of a single injection and ranged from 5 to $47 \mathrm{~m} / \mathrm{s}$. Three experimental sets were made in which one sample either received one, three or six injections. Five replicates were made for each set and solution, resulting in a total of 30 samples.

\section{Analysis}

After each topical or injection event, all samples were immediately embedded in an optimal cutting temperature mounting medium (OCT, VWR Chemicals) to stop the natural diffusion of the solutions in the skin. The samples were sectioned using a cryostat (Leica CM1950, Leica Biosystems) and analyzed under a Nikon E400 microscope for both bright field and fluorescence imaging. The solution diffusion throughout the different skin samples was quantified with the image processing software ImageJ, where the diffusion depth $\mathrm{L}_{\mathrm{M}}$ and width $\mathrm{L}_{\mathrm{T}}$ were measured $24 \mathrm{~h}$ after the topical application or injection (see Fig. 3). The applications of topical, solid or NFI themselves took 
$<10 \mathrm{~s}, 10 \mathrm{~ms}$ or $0.5 \mathrm{~ms}$ respectively (Fig. 3a). Further, the surface area of the injection site was determined with a MATLAB code developed for image processing. The fluorescence image was converted into a binary image, in which all the white pixels were added up to calculate the surface area of the injected solution (Fig. 3b). Fluorescence images of the topically applied solutions were inverted with ImageJ and overlaid with the respective bright-field images.

\section{RESULTS}

\section{Topical Application}

The dye diffusion after the topical application was first analyzed. Rhodamine B, shown in Fig. 4 in light blue, diffused $44-64 \%$ deeper into the skin than the non-fluorescing direct red 81 (pink), which remained more superficial. The direct red 81 diffusion of the glycerol solution, however, seems to be higher than in the aqueous one. Especially after $60 \mathrm{~min}$, in which the non-fluorescing part of the glycerol solution roughly diffused twice as deep as within the aqueous solution. The average diffusion depth (in $\mathrm{mm}$ ) of the fluorescing rhodamine B in both solutions was calculated from the respective samples for each time interval after 5, 30 and 60 min (see Fig. 4). In all measurements, the topical application of the glycerol solution achieved 7-29\% deeper diffusions than the aqueous one. However, using one-way ANOVA no statistically significant difference between the two solutions was identified $(p=0.34)$. The average vertical diffusion rate calculated between the three-time intervals show that the glycerol $(d z / d t=0.07 \mu \mathrm{m} / \mathrm{s})$ and aqueous solution

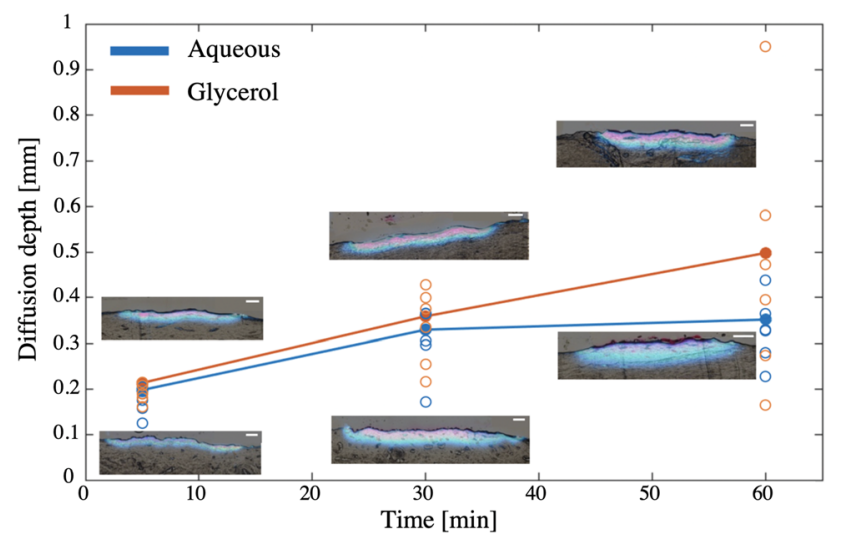

FIGURE 4. Diffusion after topical application. Average diffusion depth after 5,30 and $60 \mathrm{~min}$ of topical aqueous (blue) or glycerol (orange) solution application as shown by filled data points. Other data points represent the experimental scatter of all $\mathbf{3 0}$ samples. Inverted fluorescence (blue) and enhanced bright-field (pink) image overlay of topical diffusion. $(d z / d t=0.05 \mu \mathrm{m} / \mathrm{s})$ diffused deeper with time. For both solutions the lateral one $(d y / d t=0.2 \mu \mathrm{m} / \mathrm{s})$, however, did not change over time. The widest experimental scatter was seen with the glycerol solution after 60 min of topical application.

\section{Solid Needle Injection}

Under similar conditions as reported above, the dye dispersion of both solutions was studied after solid needle injections. The surface area of the dispersed solutions was measured with a MATLAB code and is displayed by the surface areas of the circles, as seen in Fig. 5. The averaged depth, width and area results are represented by the filled circles respectively. The dispersion depth of the glycerol solution was almost as deep as its dispersion width $(1.13 \pm 0.6 \mathrm{~mm})$. The aqueous solution, on the other hand, remained more superficial with $1.04 \pm 0.2 \mathrm{~mm}$ and instead, dispersed more in the horizontal direction $(1.28 \pm 0.3 \mathrm{~mm})$. Two fluorescent images of the respective solutions show the dispersion throughout the skin. The injection path of the solid needle into the permanently damaged tissue can be seen by the dark area in the central injection region. On average, the aqueous solution achieved a surface area dispersion of $8.6 \pm 3.2 \times 10^{3} \mathrm{~mm}^{2}$, while the glycerol solution was slightly larger with $9.2 \pm 4.8 \times 10^{-10} \mathrm{~mm}^{2}$. The terminal vertical and lateral dispersion rates for the solid needle injection are, on the one hand, determined by the maximum depth or width of each injection and, on the other hand, by the solution dispersion in the tissue. With an

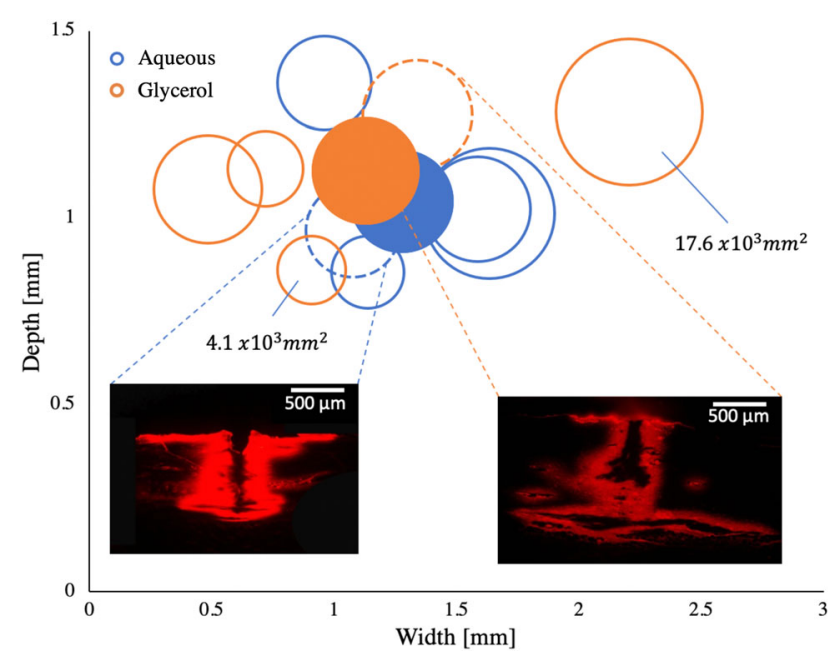

FIGURE 5. Solid needle injection. Depth and width measurements of injections with aqueous (blue) and glycerol (orange) solution. Filled circles present the average measurements respectively. The circle sizes correspond to the measured surface area of the injections. Presentation of respective fluorescence images with the same exposure time of $150 \mathrm{~ms}$. 
injection time of $20 \mathrm{~s}$, the vertical dispersion rate was $d z / d t=54 \mu \mathrm{m} / \mathrm{s}$, while the lateral one was $d y /$ $d t=60 \mu \mathrm{m} / \mathrm{s}$. The one-way ANOVA shows that there is no statistical difference between the two solutions in terms of depth $(p=0.985)$, width $(p=0.171)$ and area $(p=0.487)$.

The corresponding injection video is shown in supplementary movies.

We also performed more realistic dermal pigmentation injections in which the skin samples were set in motion along a plane perpendicular to the needle. More details of this experiment can be found in the supplementary information.

\section{Needle-Free Micro-Jet Injection}

The image sequence of an NFI, taken with $160 \times 10^{3} \mathrm{fps}$, shows one out of three single injections that were made into one porcine skin sample (Fig. 6a). The back-splash of the penetrating jet can be seen once it reached the skin (Fig. 6a bottom). Prior to the injections, the skin sample was placed at a $7.3 \mathrm{~mm}$ distance from the microfluidic device to allow visualization of the jet before impact; the jet velocity did not show any decrease up to $50 \mathrm{~cm}$ from the microfluidic device (data not shown). The first channel was partly filled with $0.03 \pm 0.003 \mu \mathrm{l}$ aqueous solution. Once the laser is triggered, a vapor bubble was created inside this channel (Fig. 6 top). The aqueous solution cavitated within $75 \pm 1.9 \mu$ s until the bubble pushes the liquid out of the first channel, while the glycerol solution needs more time $(90 \pm 1.3 \mu \mathrm{s})$ to form a jet of the same diameter $(0.1 \mathrm{~mm})$. With both solutions, however, the tip of the jets started to detach from the rest at $227 \pm 18 \mu \mathrm{s}$ of propagation. Between $329 \pm 25 \mu \mathrm{s}$, all $5.5 \pm 0.26 \mathrm{~mm}$ long jets completely left the microfluidic device. Directly after this, the jet started to break into droplets from the back of the tip in the direction of the jet displacement. The jet breakup of Newtonian liquids, such as water, are known to be caused by Rayleigh-Plateau instabilities. ${ }^{15,27}$ At that time, the jet diameter decreased to $0.09 \mathrm{~mm}$ and remained the same until the first drop of the jet hit the porcine skin surface at $468 \pm 80 \mu$ s of propagation. While the rest of the jet followed, $23.7 \pm 10.4 \%$ of the solutions splashed back. Finally, the last drop of the injected solution reached the skin surface $274 \pm 0.3 \mu$ s after the laser was triggered, while on average the injection process took $468 \pm 80 \mu$ s. The corresponding NFI video is shown in supplementary movies.

The different filters and settings of the microscope used to analyze the injected tissue allowed to take bright-field, as well as fluorescence images of the solutions. The images of the specific sample shown above are presented in Fig. 6b. The pink injection (a) time $[\mu \mathrm{s}]$

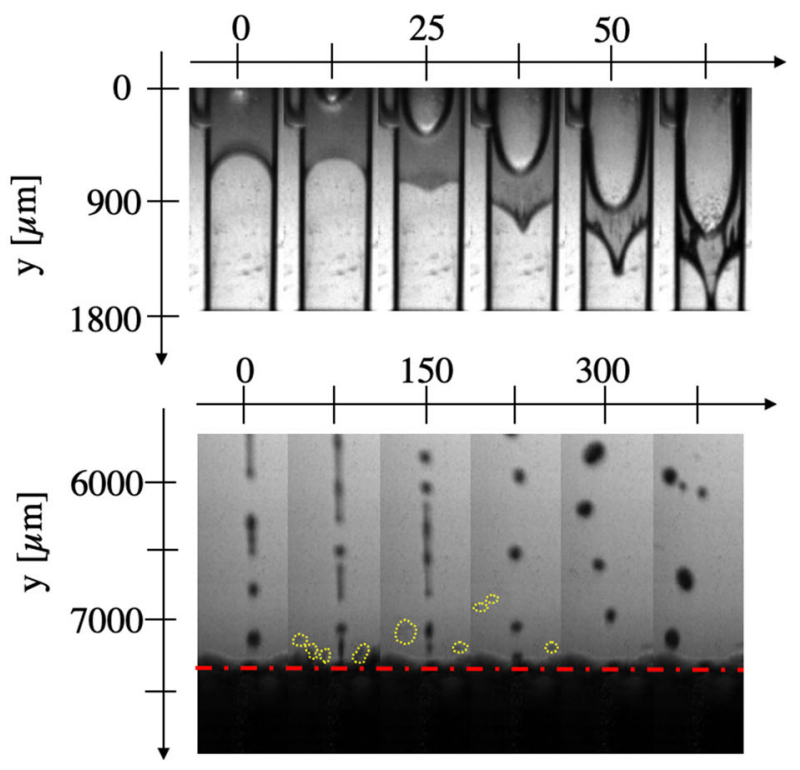

(b)
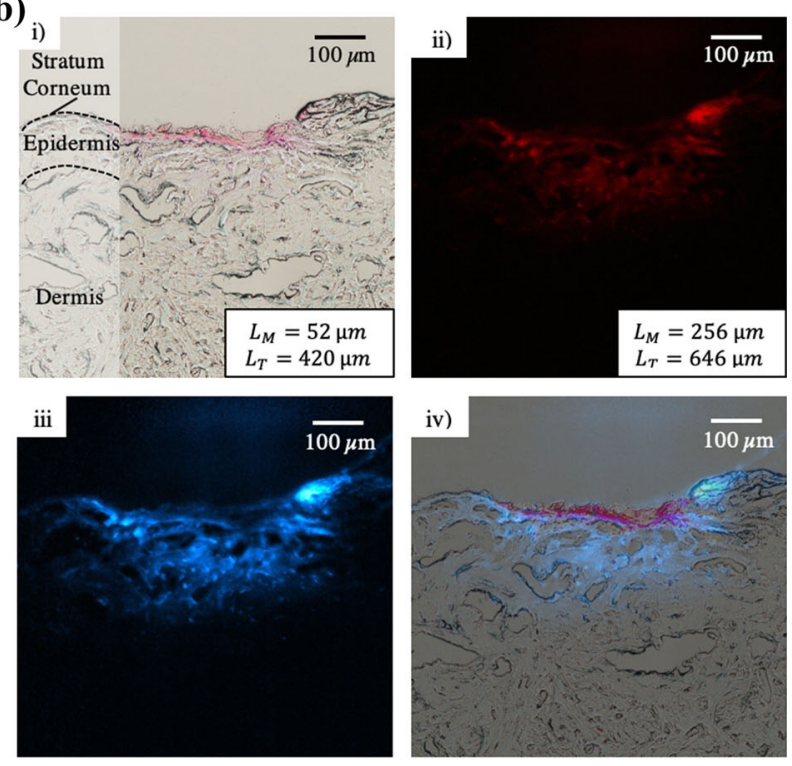

FIGURE 6. (a) Image sequences of NFI. Recordings were made at $160 \times 10^{3}$ fps. The jet velocity was $25 \mathrm{~m} / \mathrm{s}$. (Top) Bubble formation and growth by thermocavitation within $62.5 \mu \mathrm{s}$. (Bottom) Aqueous solution jet perfuses into porcine skin sample, which surface is indicated by the red dashed line. The yellow circles show the back-splashes of the jet. The distance between the microfluidic device and the skin sample is $7.3 \mathrm{~mm}$; (b) Bright-field and fluorescence images of three repetitive, NFIs with the aqueous solution. All scale bars equal to $100 \mu \mathrm{m}$. (i) Bright-field image and schematic drawing of different skin layers; (ii) Fluorescence image of solution dispersion; (iii) Fluorescence image with ImageJ modified fluorescence color afterward; (iv) Overlay of bright-field and fluorescence image.

spot could be clearly seen in the bright-field image. Its size measurements are $0.45 \mathrm{~mm}$ in width and $0.09 \mathrm{~mm}$ in depth (Fig. 6b-i). The same sample was imaged with the fluorescence settings of the micro- 


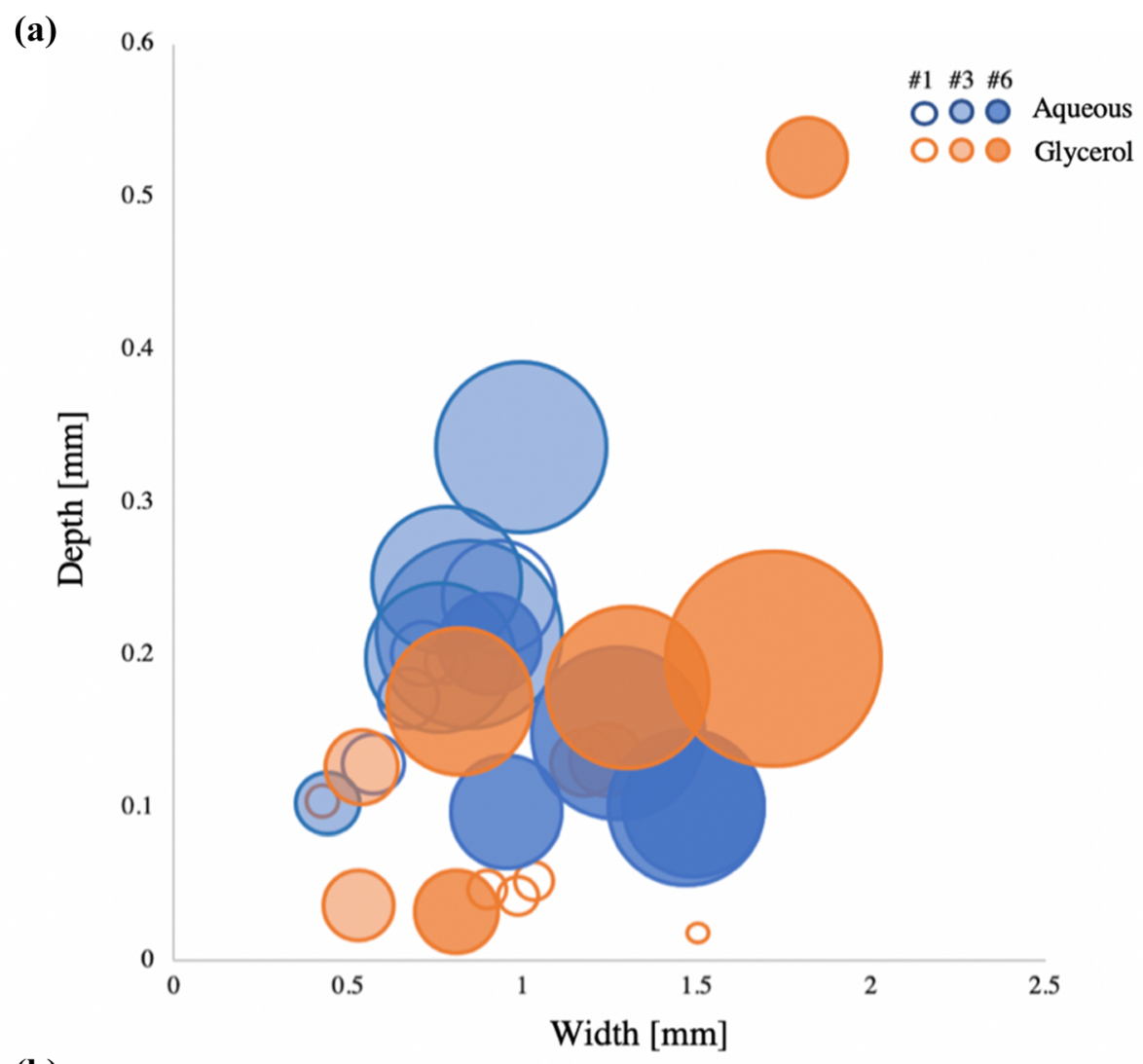

(b)

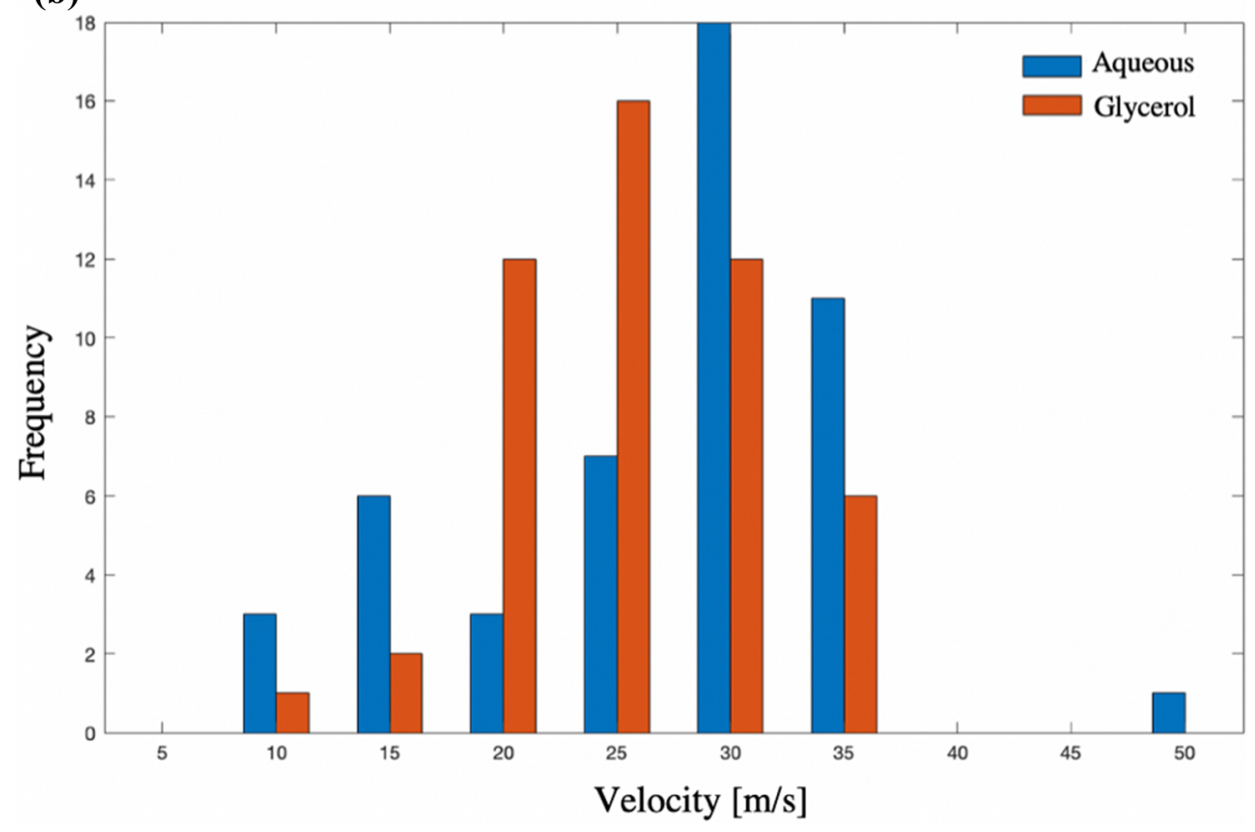

FIGURE 7. (a) Depth and width measurements of the needle-free injected solution perfusion. Perfusion depth of aqueous (blue) or glycerol (orange) solution after one (\#1), three (\#3) or six (\#6) injections. Circle sizes correspond to the measured surface area of the injections. Biggest and smallest surface areas are indicated; (b) Velocity distribution of needle-free created jets. The velocity occurrence of each aqueous (blue) and glycerol (orange) jet is plotted against the velocity.

scope. In comparison, the red area in Fig. 6b-ii) was almost three times as wide and twice as deep than the spot in the bright-field image, which becomes more evident in the overlay (Fig. 6b-iv). As for the fluorescence image of the solid needle injection, the fluorescence image had to be processed with Image J as 
well to have a higher contrast between the overlaying colors.

In the NFI experiment, the samples were differentiated by the type of injected solution and by the number of injections that were made into one sample. All data points in Fig. 7a show the size and surface measurements for samples, which received one, three or six injections. The blue data points present the injection results of the aqueous solution, while the orange ones belong to the glycerol solution. Like the solid needle measurements in Fig. 5, the circle sizes correspond to the measured surface areas of the dispersed solutions. The aqueous solution achieved greater lateral and vertical dispersions after one or three serial injections into one sample than the glycerol one. The differences of the dispersion depth, width and surface area between the solutions were less significant if six injections in a row were made into one sample. However, in all NFIs the glycerol solution resulted in a wider dispersion as in the topical application and solid needle experiments. Both terminal dispersion rates were determined as for the solid needle injection. The lateral dispersion rate $(d y /$ $d t=20 \times 10^{5} \mu \mathrm{m} / \mathrm{s}$ ) was roughly seven times bigger than its vertical one $\left(d y / d t=3 \times 10^{5} \mu \mathrm{m} / \mathrm{s}\right)$. Moreover, the velocities of all created jets are given in Fig. 7b. On average, the glycerol solution jets were $2 \mathrm{~m} / \mathrm{s}$ slower than the ones from the aqueous solution $(v=25 \pm 2.3 \mathrm{~m} / \mathrm{s})$.

\section{DISCUSSION}

\section{Aqueous and Glycerol Solutions}

After the solutions were prepared, the coloration of both observed with the naked eye was identical. However, detailed analysis with a UV-Visible spectrophotometer revealed $49 \%$ higher absorbance with the aqueous solution throughout all measured wavelengths compared to the glycerol-containing solution. For the needle-free experiments, higher laser energy absorption led to faster heating of the aqueous solution, which resulted in faster bubble expansion and higher jet velocities. For the cases of single or three injections, the aqueous solution achieved deeper dispersion than that from glycerol, since higher jet velocities have more kinetic energy to perfuse into the skin.

In all experiments and both solutions, rhodamine $B$ exhibited wider and deeper diffusion throughout the skin compared to the non-fluorescing direct red 81 . We attribute this difference to the diffusion coefficients of rhodamine $\mathrm{B}$ and direct red 81 , but contributions for lower fluorescence detection limits cannot be ruled out. In general, the solution's diffusion kinetics depend on two factors: the concentration gradient in the tissue and molecular weight. In this case, the latter factor

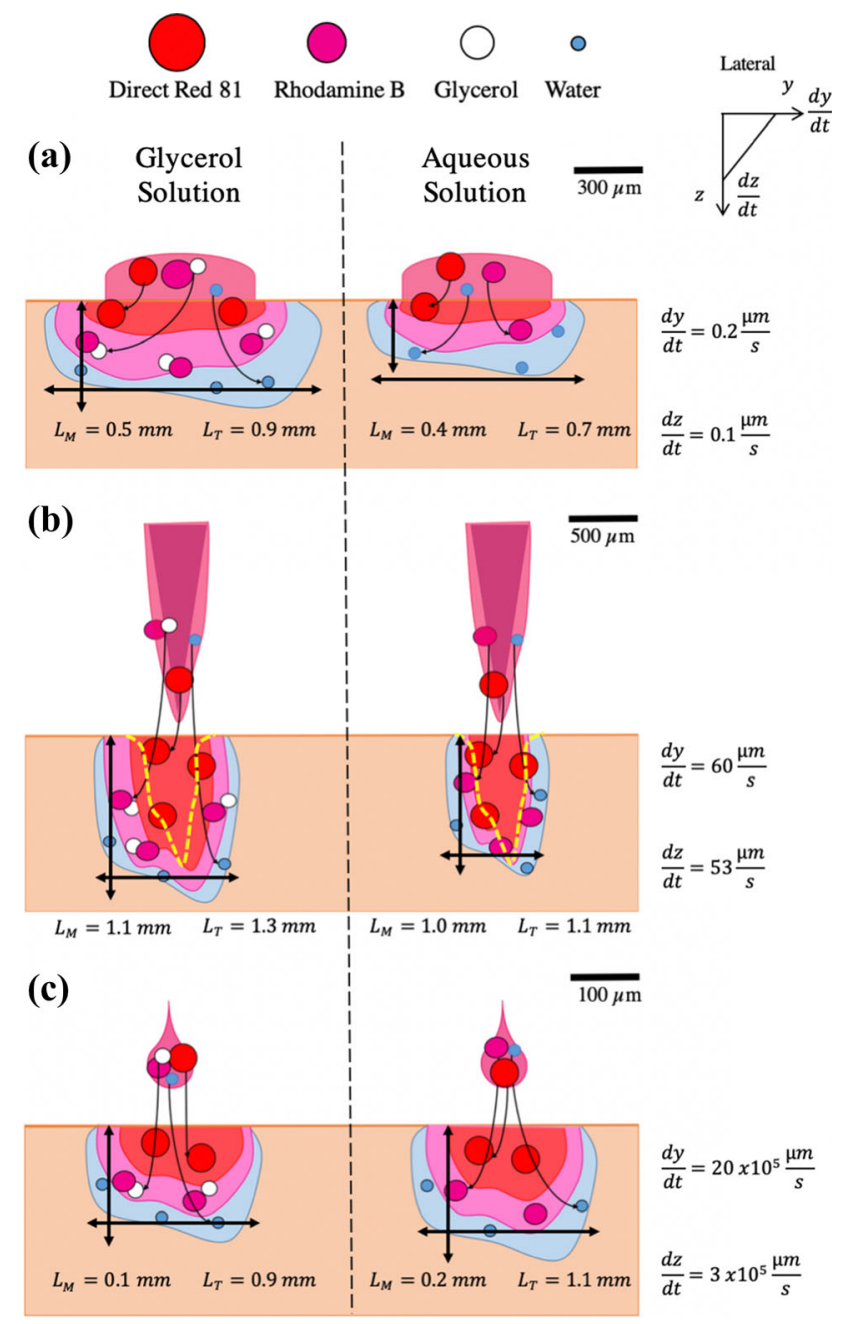

FIGURE 8. Schematic drawing of solution diffusion and dispersion in the topical application and injection methods. The left column shows the glycerol solution components diffuse or disperse differently across the skin, while the right one presents the aqueous solution. Average diffusion depth $L_{M}$ and width $L_{T}$ with the corresponding vertical, $d z / d t$, and lateral diffusion rate $d y / d t$ for the respective methods. (a) Topical application; (b) Solid needle injection. The yellow dotted line indicates the skin damage caused by needle penetration; (c) Needle-free micro-jet injection.

plays the decisive role since molecules with high molecular weights $(>500 \mathrm{~g} / \mathrm{mol})$, such as the direct red $81\left(M_{\mathrm{W}}=675.60 \mathrm{~g} / \mathrm{mol}\right)$, are known to have a poor transdermal delivery. ${ }^{5,10,38}$ The densely structured and outermost skin layer, the stratum corneum, additionally posed as a barrier for the direct red 81 macromolecules. While these were retained on the skin surface, the fluorescing rhodamine B molecules, possess a lower molecular weight $\left(M_{\mathrm{W}}=479.02 \mathrm{~g} / \mathrm{mol}\right)$ and thus, diffused more readily into the skin. The different diffusion kinetics became particularly evident if the solutions were topically applied and left on the skin for a longer diffusion time. The $7-29 \%$ deeper diffusion depths of the glycerol solution compared to 
the aqueous solution, however, are due to the different diffusion coefficients $D .^{11,43}$

\section{Comparison of Transdermal Delivery Methods}

Topical application is the easiest and possibly the least invasive way to deliver molecules into the body. As mentioned before, the skin barrier limits this passive method to lipophilic molecules with a low molecular weight. Depositing and removing the solution droplet in the same time scale as the NFI or solid needle would not have been possible (within ms) $f$ or a fair comparison. To overcome the SC barrier limitation, we extended the experimental time up to $60 \mathrm{~min}$ and still only achieved diffusion depths of $0.35 \mathrm{~mm}$ or $0.5 \mathrm{~mm}$ with the aqueous or glycerol solution. During the same time, 10 or 60 skin injections could have been made with the needlefree or solid needle method respectively. The calculated diffusion rate in vertical direction, $d z / d t=0.1 \mu \mathrm{m} / \mathrm{s}$, was the slowest of all compared methods (Fig. 8a).

The solid needle injections with the PMU machine achieved the deepest penetration with a vertical penetration rate $d z / d t=54 \mu \mathrm{m} / \mathrm{s}$. However, it was also the most invasive method. As seen in Fig. 8b, needle penetration is detrimental to skin integrity, and is known to pose risks of infection and scarring. ${ }^{5,12}$ The bright-field and fluorescence images show the ruptured tissue, which was caused by the needle penetration. However, this tissue damage did not always correspond to a deep dispersion depth of the solution. In terms of the experimental procedure, the adjustable PMU pen holder enabled a quick and repeatable injection process for multiple samples with the same penetration depth.

The injections with the NFI device are 20 times faster and lead to lateral and vertical diffusion rates larger than the solid injector, ca. 30,000 and 6000-fold respectively (Fig. 8c). The preparations for jet injections take more time since this setup is still experimental (30 min longer). Defects within the microfluidic devices, which appear during the production process, and gas bubbles that are formed in the channel if filled improperly, can scatter the incoming laser light. Less energy is absorbed by the liquid and as a consequence, the vapor bubble within the device does not grow rapidly enough to displace the liquid out of the channel. The image sequence in Fig. 6a-ii) shows that $6 \%(1.8 \mathrm{~nL})$ of the solution jet splashed back from the skin surface. On average, $23.7 \pm 10.4 \%$ of the initial filling volumes did not perfuse into the skin due to splashbacks. According to the literature, a minimum jet velocity of $13 \mathrm{~m} / \mathrm{s}$ should be sufficient to penetrate a typical skin strength of $20 \mathrm{MPa}{ }^{8,36}$ Out of all created jets, $92 \%$ were faster or even twice as fast than this velocity.

The most relevant observation is that no tissue damage was observed after analyzing the cross-section images. The stratum corneum remained intact even after six repetitive injections into the same sample spot. Since all excessive solutions were carefully removed from the skin after each injection and before freezing, we can assume that passive diffusion plays a negligible role. A comparison of the dispersion kinetics shows that the NFI method is three to six orders of magnitude higher than the solid needle and topical one respectively. In theory, the needle-free micro-jet injector is more efficient in terms of dispersion depth per second than the traditional methods. However, multiple injections with the needle-free device have shown that the solution dispersion does not increase linearly in time or number of injections. Further studies are required to investigate the actual dependency between a given number of injection events and the corresponding final dispersion patterns.

We calculated the kinetic energy $K=1 / 2 \mathrm{mv}^{2}$ of the needle and liquid jet that is transferred to the skin and evaluate the injection efficiency in terms of energy per injection $\varepsilon_{\text {energy }}=K / E \times 100 \%$ (Table 1). The PMU

TABLE 1. Comparison between the solid needle and needle-free micro-jet methods.

\begin{tabular}{|c|c|c|c|c|c|c|c|c|c|c|}
\hline \multirow[b]{2}{*}{ Method } & \multirow[b]{2}{*}{ Solution } & \multirow[b]{2}{*}{$p(\mathrm{kPA})$} & \multirow[b]{2}{*}{$S=p / E^{b}$} & \multirow[b]{2}{*}{$K(\mu \mathrm{J})$} & \multirow[b]{2}{*}{$\varepsilon_{\text {Energy }} \%$} & \multicolumn{3}{|c|}{ First injection } & \multicolumn{2}{|c|}{ End point $^{a}$} \\
\hline & & & & & & $L(\mathrm{~mm})$ & $D(\mathrm{~mm})$ & $\varepsilon_{\mathrm{vol}} \% V_{0}(\mathrm{~nL})$ & $L(\mathrm{~mm})$ & $D(\mathrm{~mm})$ \\
\hline \multirow[t]{2}{*}{ Solid needle } & Glycerol & 2400 & 4.8 & 67.11 & 95.88 & N.A. & N.A. & $\begin{array}{c}4.37 \\
351.55\end{array}$ & 0.81 & 0.27 \\
\hline & Aqueous & & & & & N.A. & N.A. & $\begin{array}{c}1.90 \\
357.27\end{array}$ & 0.90 & 0.17 \\
\hline \multirow[t]{2}{*}{ Needle-free injection } & Glycerol & 270 & 0.54 & 11.71 & 10.84 & 0.05 & 0.97 & $\begin{array}{l}75.06 \\
38.50\end{array}$ & 0.22 & 1.29 \\
\hline & Aqueous & 304 & 0.61 & 9.73 & 10.82 & 0.19 & 0.73 & $\begin{array}{l}90.31 \\
32.00\end{array}$ & 0.13 & 1.22 \\
\hline
\end{tabular}

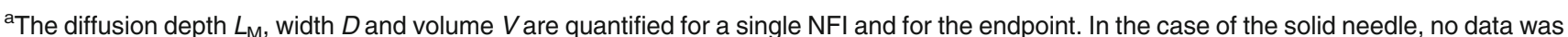
available for one injection since the shortest injection time within these experiments were $20 \mathrm{~s}$. For the solid needle 200 injections were performed, whereas six jet injections were made.

${ }^{\mathrm{b}}$ The ratio between the injection pressure $p$ and the skin's Young's modulus $E_{S k i n}=0.5 \mathrm{MPa},{ }^{7}$ defines the penetration strength $S$. 
machine has six and nine times higher $K$ and $\varepsilon_{\text {energy }}$ respectively than the NFI device because of the larger mass. The penetration strength $S=p / E$ indicates potential skin damage by quantifying the skin rupture and penetration characteristics. This dimensionless variable is the strength ratio between the injection pressure $p$ exerted onto the skin and tabulated skin's Young's modulus $E_{\text {Skin }}=0.5 \mathrm{MPa}^{7}$ The jet pressure is calculated as $p_{\text {jet }}=1 / 2 \rho v_{\text {jet }}^{2}$ with $\rho$ as the liquid density and $v_{\text {jet }}$ as the jet velocity. ${ }^{44}$ The needle pressure value $(p=2400 \mathrm{kPa})$, taken from a similar experiment with agarose, is assumed to be within the same order of magnitude for porcine skin. ${ }^{33}$ Both solutions in the NFI experiments resulted in a nine times lower penetration strength than the solid needle $(S=4.8)$. Despite their low $p$ and thus, $S$ values $(S<1)$, which theoretically implies no perfusion, the bright-field and fluorescence images confirm the jets entered the skin. ${ }^{45}$ We hypothesize that the Young's modulus of these skin samples is lower than obtained from literature, since the moduli vary for different skin samples and are highly dependent on the anatomical skin location and measurement technique, such as stress-strain or indenting experiments. ${ }^{1,7,14,50}$ As for the efficiency of volume injected per injection event, the ejected (NFI) or deposited volume (solid needle) $V_{0}$ is set in relation with the volume remaining in the skin as $\varepsilon_{\mathrm{vol}}=V_{\mathrm{inj}} / V_{0} \times 100 \%$. The following volume estimations are supposed to be taken as an indication, while future studies should focus on more precise measurements. The $\varepsilon_{\mathrm{vol}}$ of the NFI device was 19-45-fold higher than the PMU machine since the solid needle only injected $2-4 \%$ of its initial filling volume into the skin. The NFI device, on the other hand, injected $75-90 \%$ of its volume. The methods used to obtain these values are described in more detail elsewhere. ${ }^{33}$

In real-life conditions, repeatability or reproducibility of multiple injections may require that the jet injection frequency is increased to larger values than possible with the current experimental setup. To assess the dispersion patterns within the in vitro and in vivo skin samples, optical coherence tomography (OCT) can be used to calculate the transfected volume since current 2D images are too asymmetric to revolve the calculated area around the axis of symmetry. ${ }^{13}$ For future in vivo studies, we consider that the temperature of the liquid jets has to be measured to assess any probable burn injuries of the skin. To this aim, a thermographic high-speed camera could be used. As a next step, the caused pain needs to be evaluated in in vivo studies, as it can be rated subjectively by each individual. Further, for future injections of medications, the molecular structure of the medication is not expected to change due to laser exposure and thermocavitation. The UV-visible spectrophotometer and proton nuclear magnetic resonance $\left({ }^{\mathrm{H}} \mathrm{NMR}\right)$ could be used to detect any structural changes and by-product generation due to heat.

The comparison of three transdermal delivery methods based on topical application, solid needle and, needle-free micro-jet injection, has shown that the latter was able to achieve a dispersion depth comparable to the topical application. The needle-free microjet injection has a faster injection and higher dispersion rate, which makes it a more efficient transdermal delivery method than the traditional ones. Moreover, no tissue damage was observed unlike the results with a solid needle injector. Since the needle-free micro-jet injector is still in an early development phase, experiments with human skin are needed to investigate if the created liquid jet is able to perfuse it causing minimal damage. In theory, a needle-free injection might perfuse less deep into human than porcine skin due to the different and larger Young's moduli of the stratum corneum. ${ }^{25,40,41}$ It is assumed that the penetration depth of the solid needle injection is not affected due to its high penetration strength $\mathrm{S}$, which is defined by the ratio between injection pressure and Young's modulus. Since we did not observe damage to the skin with the jet injector, future studies should be focused on finding out if the injectate transfects the tissue via the intercellular space, or through cells.

It can be concluded that the needle-free micro-jet injector has the potential to improve drug delivery across the skin if the challenges mentioned above are overcome. Millions of people that currently use painful solid needles for medical or cosmetic applications could soon replace them with a less invasive and safer liquid injection method.

\section{ELECTRONIC SUPPLEMENTARY MATERIAL}

The online version of this article (https://doi.org/10. 1007/s10439-019-02383-1) contains supplementary material, which is available to authorized users.

\section{ACKNOWLEDGMENTS}

We would like to thank Dr. Loreto Oyarte-Galvez for her assistance with experiments, Stefan Schlautmann and Frans Segerink for their technical support with the microfluidic devices and the optical setup construction. The material support of PERMANENTLine $\mathrm{GmbH}$ Co. KG. and MT-Derm is kindly 
acknowledged. DFR acknowledges the recognition from the Royal Dutch Society of Sciences (KHMW) that granted the Pieter Langerhuizen Lambertuszoon Fonds, 2016.

\section{OPEN ACCESS}

This article is distributed under the terms of the Creative Commons Attribution 4.0 International License (http://creativecommons.org/licenses/by/4.0/), which permits unrestricted use, distribution, and reproduction in any medium, provided you give appropriate credit to the original author(s) and the source, provide a link to the Creative Commons license, and indicate if changes were made.

\section{REFERENCES}

${ }^{1}$ Agache, P. G., C. Monneur, J. L. Leveque, and J. De Rigal. Mechanical properties and young's modulus of human skin in vivo. Arch. Dermatol. Res. 269(3):221-232, 1980.

${ }^{2}$ American Psychiatric Association. DSM-IV Sourcebook, Vol. 1. Washington DC: American Psychiatric Publishing, 1994.

${ }^{3}$ Archer, D. F., V. Cullins, G. W. Creasy, and A. C. Fisher. The impact of improved compliance with a weekly contraceptive transdermal system (ortho evra ${ }^{\circledR}$ ) on contraceptive efficacy. Contraception 69(3):189-195, 2004.

${ }^{4}$ Arora, A. Liquid and powder jet injectors in drug delivery: Mechanisms, designs, and applications. In: Percutaneous Penetration Enhancers Physical Methods in Penetration Enhancement, edited by N. Dragicevic, and H. I. Maibach. Berlin: Springer, 2017, pp. 221-230.

${ }^{5}$ Arora, A. M., R. Prausnitz, and S. Mitragotri. Micro-scale devices for transdermal drug delivery. Int. J. Pharm. 364(2):227-236, 2008.

${ }^{6}$ Bardal, S., J. Waechter, and D. Martin. Applied Pharmacology. e-Book. Amsterdam: Elsevier Health Sciences, 2010.

${ }^{7}$ Baxter, J., and S. Mitragotri. Jet-induced skin puncture and its impact on needle-free jet injections: Experimental studies and a predictive model. J. Control. Release. 106(3):361-373, 2005.

${ }^{8}$ Berrospe-Rodriguez, C., C. W. Visser, S. Schlautmann, R. Ramos-Garcia, and D. Fernandez Rivas. Continuouswave laser generated jets for needle free applications. Biomicrofluidics 10:014104, 2016.

${ }^{9}$ Berrospe-Rodriguez, C., C. W. Visser, S. Schlautmann, D. Rivas, and R. Ramos-Garcia. Toward jet injection by continuous-wave laser cavitation. J. Biomed. Opt. 22(10):105003, 2017.

${ }^{10}$ Brown, M. B., G. P. Martin, S. A. Jones, and F. K. Akomeah. Dermal and transdermal drug delivery systems: Current and future prospects. Drug Deliv. 13:175-187, 2006.

${ }^{11}$ Brunner, G. Hydrothermal and Supercritical Water Processes, Vol. 5. Amsterdam: Elsevier, 2014.

${ }^{12}$ Chase, C. C., C. S. Daniels, R. Garcia, F. Milward, and T. Nation. Needle-free injection technology in swine: Progress toward vaccine efficacy and pork quality. J. Swine Health Prod. 16(5):254-261, 2008.

${ }^{13}$ Coulman, S. A., et al. In vivo, in situ imaging of microneedle insertion into the skin of human volunteers using optical coherence tomography. Pharm. Res. 28(1):66-81, 2011.

${ }^{14}$ Daly, C. H. Biomechanical properties of dermis. J. Invest. Dermatol. 79:17-20, 1982.

${ }^{15}$ Eggers, J., and E. Villermaux. Physics of liquid jets. Rep. Prog. Phys. 71:036601, 2008.

${ }^{16}$ Fluhr, J., R. Darlenski, and C. Surber. Glycerol and the skin: Holistic approach to its origin and functions. $\mathrm{Br} . J$. Dermatol. 159:23-34, 2008.

${ }^{17}$ Hamilton, J. G. Needle phobia: A neglected diagnosis. $J$. Fam. Pract. 41(2):169-175, 1995.

${ }^{18}$ Hogan, N. C., A. J. Taberner, L. A. Jones, and I. W. Hunter. Needle-free delivery of macromolecules through the skin using controllable jet injectors. Expert Opin. Drug Deliv. 12(10):1637-1648, 2015.

${ }^{19}$ Jang, H. J., S. Yeo, and J. J. Yoh. Synchronization of skin ablation and microjet injection for an effective transdermal drug delivery. App. Phys. A 122(4):320, 2016.

${ }^{20}$ Jarupanich, T., S. Lamlertkittikul, and V. Chandeying. Efficacy, safety and acceptability of a seven-day, transdermal estradiol patch for estrogen replacement therapy. $J$. Med. Assoc. Thai. 86:836-845, 2003.

${ }^{21}$ Kim, Y.-C. Skin vaccination methods: Gene gun, jet injector, tattoo vaccine, and microneedle. In: Percutaneous Penetration Enhancers Physical Methods in Penetration Enhancement, edited by N. Dragicevic, and H. I. Maibach. Berlin: Springer, 2017, pp. 485-499.

${ }^{22}$ Kiyama, A., et al. Visualization of penetration of a highspeed focused microjet into gel and animal skin. J. Vis. 22(3):449-457, 2019.

${ }^{23}$ Kluger, N., J. Serup, and W. Bäumler (eds.). Current Problems in Dermatology, Vol. 48. Tattooed Skin and Health, Basel: Karger, pp. 21-30, 2015.

${ }^{24}$ Kwon, K. M., S.-M. Lim, S. Choi, D.-H. Kim, H.-E. Jin, G. Jee, K.-J. Hong, and J. Y. Kim. Microneedles: Quick and easy delivery methods of vaccines. Clin. Exp. Vaccine Res. 6:156-159, 2017.

${ }^{25}$ Lavker, R. M., G. Dong, P. Zheng, and G. F. Murphy. Hairless micropig skin. A novel model for studies of cutaneous biology. Am. J. Pathol. 138(3):687-697, 1991.

${ }^{26} \mathrm{Li}, \mathrm{X}$., B. Ruddy, and A. Taberner. Characterization of needle-assisted jet injections. J. Control. Release. 243:195203, 2016.

${ }^{27}$ Lord Rayleigh, F. R. S. On the instability of jets. P Lond Math Soc 1(1):4-13, 1878.

${ }^{28}$ Mckeage, J. W., B. P. Ruddy, P. M. Nielsen, and A. J. Taberner. The effect of jet speed on large volume jet injection. J. Control Release 280:51-57, 2018.

${ }^{29}$ Menezes, Kumar. Takayama "Shock wave driven liquid microjets for drug delivery". J. Appl. Phys. 106:086102, 2009.

${ }^{30}$ Mitchell, H., T. Hamilton, F. Steggerda, and H. Bean. The chemical composition of the adult human body and its bearing on the biochemistry of growth. J. Biol. Chem. 158:625-637, 1945.

${ }^{31}$ Mitragotri, S. Current status and future prospects of needle-free liquid jet injectors. Nat. Rev. Drug Discov. 5:543548, 2006.

${ }^{32}$ Münch, S., J. Wohlrab, and R. Neubert. Dermal and transdermal delivery of pharmaceutically relevant macromolecules. Eur. J. Pharm. Biopharm. 119:235-242, 2017. 
${ }^{33}$ Oyarte Gálvez, L., M. Brió Pérez, and D. Fernández Rivas. High speed imaging of solid needle and liquid micro-jet injections. AIP J. Appl. Phys. 125:144504, 2019.

${ }^{34}$ Padilla-Martinez, J., et al. Towards the enhancement of transdermal drug delivery through thermocavitation. Photonics Lasers Med. 1(3):183-193, 2012.

${ }^{35}$ Padilla-Martinez, J., C. Berrospe-Rodriguez, G. Aguilar, J. Ramirez-San-Juan, and R. Ramos Garcia. Optic cavitation with cw lasers: A review. Phys. Fluids 26:122007, 2014.

${ }^{36}$ Park, M. A., H. J. Jang, F. V. Sirotkin, and J. J. Yoh. Er:YAG laser pulse for small-dose splashback-free microjet transdermal drug delivery. Opt. Lett. 37(18):3894-3896, 2012.

${ }^{37}$ Payne, R., S. D. Mathias, D. J. Pasta, L. A. Wanke, R. Williams, and R. Mahmoud. Quality of life and cancer pain: Satisfaction and side effects with transdermal fentanyl versus oral morphine. J. Clin. Oncol. 16:1588-1593, 1998.

${ }^{38}$ Prausnitz, M. R., and R. Langer. Transdermal drug delivery. Nat. Biotechnol. 26(11):1261-1268, 2008.

${ }^{39}$ Prausnitz, M. R., S. Mitragotri, and R. Langer. Current status and future potential of transdermal drug delivery. Nat. Rev. Drug. Discov. 3(2):115-124, 2004.

${ }^{40}$ Ranamukhaarachchi, S. A., et al. A micromechanical comparison of human and porcine skin before and after preservation by freezing for medical device development. Sci. Rep. 6:32074, 2016.

${ }^{41}$ Ranamukhaarachchi, S. A., et al. Development and validation of an artificial mechanical skin model for the study of interactions between skin and microneedles. Macromol. Mater. Eng. 301(3):306-314, 2016.

${ }^{42}$ Rastopov, S. F., and A. T. Sukhodolsky. Sound generation by thermocavitation-induced $\mathrm{cw}$ laser in solutions. In: Optical Radiation Interaction with Matter, Vol. 1440, edited by A. M. Bonch-Bruevich, V. I. Konov, and M. N. Libenson. Bellingham: International Society for Optics and Photonics, 1991, pp. 127-135.

${ }^{43}$ Rousseau, R. W. Handbook of Separation Process Technology. Hoboken: Wiley, 1987.

${ }^{44}$ Schramm, J., and S. Mitragotri. Transdermal drug delivery by jet injectors: Energetics of jet formation and penetration. Pharm. Res. 19(11):1673-1679, 2002.

${ }^{45}$ Seok, J., C. T. Oh, H. J. Kwon, T. R. Kwon, E. J. Choi, S. Y. Choi, S. K. Mun, S. H. Han, B. J. Kim, and M. N. Kim. Investigating skin penetration depth and shape following needle-free injection at different pressures: A cadaveric study. Lasers Surg. Med. 48(6):624-628, 2016.

${ }^{46}$ Simmons, J., et al. Characterization of skin blebs from intradermal jet injection: Ex vivo studies. J. Control Release 307:200-210, 2019.

${ }^{47}$ Taberner, A., N. C. Hogan, and I. W. Hunter. Needle-free jet injection using real- time controlled linear lorentz-force actuators. Med. Eng. Phys. 34(9):1228-1235, 2012.

${ }^{48}$ Tagawa, Y., et al. Highly focused supersonic microjets. Phys. Rev. X 2.3:031002, 2012.

${ }^{49}$ Tagawa, Y., et al. Needle-free injection into skin and soft matter with highly focused microjets. Lab Chip 13:13571363, 2013.

${ }^{50}$ Żak, M., P. Kuropka, M. Kobielarz, A. Dudek, K. KaletaKuratewicz, and S. Szotek. Determination of the mechanical properties of the skin of pig foetuses with respect to its structure. Acta Bioeng. Biomech. 13(2):37-43, 2011.

Publisher's Note Springer Nature remains neutral with regard to jurisdictional claims in published maps and institutional affiliations. 